\title{
Estudo da correlação entre pressão intra-ocular e espessura corneana central (projeto glaucoma)
}

\author{
Intraocular pressure vs central corneal thickness: \\ a comparative study (glaucoma project)
}

\author{
Kenji Sakata ${ }^{(1)}$ \\ Aloisio Laurindo de M. Figueira ${ }^{(2)}$ \\ Ana Cecília Pedriali Guimarães ${ }^{(3)}$ \\ Artur José Schmitt ${ }^{(4)}$ \\ Luciana Scapucin ${ }^{(5)}$ \\ Luis Guilherme Reg'o Barros ${ }^{(6)}$ \\ Nara Delai ${ }^{(7)}$
}

\section{RESUMO}

Objetivos: Avaliar a distribuição da espessura corneana central (ECC) e sua relação com a pressão intra-ocular (PIO) em um grupo de pacientes e demonstrar a utilidade da paquimetria para avaliar a PIO em casos selecionados.

Métodos: A espessura corneana central foi determinada em 167 pacientes com mais de 40 anos de idade (319 olhos) por meio de paquimetria ultra-sônica. A pressão intra-ocular foi medida com tonômetro de aplanação de Goldmann.

Resultados: A ECC média dos 319 olhos foi $0,5173+0,0377 \mathrm{~mm}$, sendo o valor máximo 0,656 mm e o mínimo 0,430 mm. A PIO média foi $16,44+3,88 \mathrm{mmHg}$, a pressão máxima $30 \mathrm{mmHg}$ e a pressão mínima 8 mmHg. Foi observada uma regressão linear de 0,13677 nas variáveis analisadas $(p=0,0145)$, não havendo diferença entre sexo e idade. Dividiu-se as observações em dois grupos: grupo I - PIO $\leq 21$ mmHg - com 285 olhos que apresentaram uma ECC média de 0,517 + 0,0376; e o grupo II - PIO > 21 mmHg - com 34 olhos que apresentaram uma ECC média de $0,519+0,0393$.

Conclusão: Observou-se uma regressão linear entre PIO e ECC, ou seja, quanto maior a ECC maior será a PIO. Demonstrou-se a utilidade da paquimetria corneana na avaliação da PIO daqueles pacientes em que esta estava falsamente aumentada ou diminuída na tonometria de aplanação, direcionando a terapêutica a pacientes realmente portadores de glaucoma.

Palavras-chave: Pressão intra-ocular; Espessura corneana central.
Trabalho realizado tendo como base dados provenientes do "Projeto Glaucoma" - Projeto de Extensão Universitária - UFPR. O Projeto Glaucoma conta com o apoio de: Rotary Club - Cristo Rei; Laboratórios Allergan-Frumtost; Pró-Reitoria de Extensão e Cultura - UFPR.

Cada autor declara que ele/ela não possui interesse financeiro no desenvolvimento ou marketing dos (instrumentos, medicação) referidos no estudo.

(1) Responsável pelo Setor de Glaucoma do Departamento de Oftalmo-Otorrinolaringologia do Hospital das Clínicas - UFPR.

${ }^{(2,3,4)}$ Acadêmicos do $6^{\circ}$ ano do Curso de Medicina-UFPR.

(5) Residente do $1^{\circ}$ ano de Oftalmologia do Hospital das Clínicas - UFPR.

(6,7) Residentes do $2^{\circ}$ ano de Oftalmologia do Hospital das Clínicas - UFPR.

Endereço para correspôndencia: Dr. Kenji Sakata R. Itupava, 1428. Alto da Rua XV. Curitiba (PR) CEP 80040-000 e-mail: sakata@vrsystems.com.br

\section{INTRODUÇ̃̃̃O}

A pressão intra-ocular (PIO) é utilizada no diagnóstico e controle de muitas patologias oculares, incluindo os diversos tipos de glaucoma. $\mathrm{O}$ conceito de glaucoma modificou-se muito nos últimos anos. Atualmente considera-se o glaucoma como uma neuropatia óptica em que a PIO é o seu maior fator de risco e não sua única e exclusiva causa ${ }^{1}$. Existem vários aparelhos (tonômetros) que são destinados a medir a PIO, sendo os principais: os tonômetros de indentação, os tonômetros de não contato (sopro de ar) e os tonômetros de aplanação. Dentre estes, o mais utilizado é o tonômetro de aplanação de Goldmann, por ser um método prático e confiável de medida da $\mathrm{PIO}^{1,2}$.

Existem vários fatores que influenciam os níveis da PIO (variação diurna, variação postural, movimentos de pálpebras e do olho, influência 
hormonal e alimentos) e a medida destes níveis (alterações na curvatura mediana corneana e espessura corneana) ${ }^{1,3,4}$. A tonometria de aplanação de Goldmann é o padrão ouro de medida, mas fornece somente uma estimativa da PIO ${ }^{5,6}$. A precisão depende da utilização de uma técnica correta, dependendo sobretudo da utilização de uma quantidade adequada de fluoresceína ${ }^{7}$. Entretanto, erros dependentes da espessura e rigidez corneanas alteradas não podem ser evitados através da utilização deste método ${ }^{5,6}$, já que o tonômetro de Goldmann é calibrado para uma espessura corneana central (ECC) de $0,54 \mathrm{~mm}$, o que sugere que espessuras acima deste valor poderiam teoricamente alterar a mensuração ${ }^{20}$, resultando em valores falsamente aumentados, enquanto espessuras menores resultariam em valores falsamente diminuídos ${ }^{5-10}$.

A ECC pode ser medida por métodos ópticos ou através da ultra-sonografia, sendo o último mais confiável ${ }^{5,8}$.

O presente estudo visa avaliar a distribuição da ECC e sua relação com a pressão intra-ocular num grupo de pacientes adultos e demonstrar a utilidade da paquimetria corneana no esclarecimento de PIO falsamente aumentada ou falsamente diminuída à tonometria de aplanação, a fim de estabelecer terapêutica adequada aos pacientes realmente glaucomatosos.

\section{PACIENTES E MÉTODOS}

Um estudo prospectivo observacional foi realizado de Julho a Outubro de 1998 no Ambulatório de Oftalmologia do Hospital de Clínicas da UFPR. A população avaliada era composta por moradores de asilos e casas de repouso localizadas na cidade de Curitiba.

A população estudada compreendeu um total de 167 pacientes (319 olhos), com idade a partir de 40 anos e de ambos os sexos. Foram excluídos da amostra os olhos que possuíam história pregressa de cirurgia ocular, phtisis bulbi, miras irregulares à tonometria de aplanação ou astigmatismo maior que 4 dioptrias (dp), pois sabe-se que astigmatismos regulares com diferenças maiores ou iguais a $4 \mathrm{dp}$ podem alterar em até $1 \mathrm{mmHg}$ a medida da PIO ${ }^{1,11}$.

Todos os olhos tiveram a pressão intra-ocular aferida através do tonômetro de aplanação de Goldmann, modelo "Haag-Streit", e a espessura corneana central (ECC) mensurada através do paquímetro ultra-sônico da marca Topcon modelo SP-2000P. Todos os olhos foram submetidos a fundoscopia com oftalmoscópio direto e binocular indireto.

Foram considerados valores normais de PIO aqueles inferiores ou iguais a $21 \mathrm{~mm} \mathrm{Hg}^{1,5}$. Com relação à ECC adotouse como normal um valor médio de $0,54 \mathrm{~mm}^{1,13}$.

A medida da PIO era realizada da seguinte maneira: colocava-se o paciente sentado e instilava-se 1 gota de colírio anestésico e fluoresceína $10 \%$ em fundo de saco conjuntival, aguardando-se um tempo médio de 10 segundos para se realizar a aferição da PIO. Esta aferição era feita através do toque dos bordos internos dos semicírculos formados ao se tocar o biprisma suavemente no centro da córnea, utilizando- se a luz azul de cobalto. Miras irregulares eram excluídas do estudo. Foram realizadas três medidas da PIO em cada olho, realizadas por um mesmo examinador, sendo utilizada para o estudo a média calculada.

Os pacientes com PIO maior que $21 \mathrm{mmHg}$ ou alterações fundoscópicas suspeitas de glaucoma eram encaminhados a consultas subsequentes para realização de gonioscopia e campo visual, sendo feito tratamento dos pacientes com diagnóstico confirmado de glaucoma.

Os pacientes foram divididos em dois grupos: pacientes com PIO maior que $21 \mathrm{mmHg}$ e pacientes com PIO menor ou igual. Correlacionou-se então, a média mais desvio padrão das PIO com a média mais desvio padrão das ECC através do método estatístico de regressão linear.

\section{RESULTADOS}

Conforme observamos na tabela 1 , a média da espessura corneana central dos 319 olhos avaliados foi de 0,5173 \pm $0,0377 \mathrm{~mm}$, sendo os valores máximo e mínimo encontrados 0,656 e $0,430 \mathrm{~mm}$ respectivamente.

A média da PIO foi de $16,44 \pm 3,88 \mathrm{mmHg}$, sendo a pressão máxima obtida de 30 e a mínima de $8 \mathrm{mmHg}$ (tabela 1 ).

Dentre os olhos avaliados, 285 apresentavam PIO $\leq 21$ $\mathrm{mmHg}$, sendo a média da ECC 0,517 $\pm 0,0376 \mathrm{~mm}$, com valor mínimo de 0,430 e máximo de $0,660 \mathrm{~mm}$. Os 34 olhos restantes apresentavam $\mathrm{PIO}>21 \mathrm{mmHg}$, com uma média da ECC de $0,519 \pm 0,0393 \mathrm{~mm}$, sendo o valor mínimo de 0,440 e o máximo de $0,620 \mathrm{~mm}$ (tabela 2 ).

Observou-se uma correlação linear de 0,13677 entre as variáveis analisadas $(\mathrm{p}=0,0145)$, ou seja, quanto maior a espessura corneana central mais alta a pressão intra-ocular. (gráfico 1)

\section{DISCUSSÃO}

O valor de normalidade de PIO é baseado nos estudos de Leydhecker e cols ${ }^{12}$ onde consideram-se normais pressões de 15,5 $\pm 2,6 \mathrm{mmHg}$, aceitando-se como limites de normalidade

\begin{tabular}{|llllc|}
\hline \multicolumn{4}{|c|}{ Tabela 1. Valores PIO e ECC de todas as observações } \\
$\mathbf{n = 3 1 9}$ & Média & $\begin{array}{c}\text { Desvio } \\
\text { padrão }\end{array}$ & V. máx. & V. mín. \\
PIO & $16,44 \mathrm{mmHg}$ & $3,88 \mathrm{mmHg}$ & $30 \mathrm{mmHg}$ & $8 \mathrm{mmHg}$ \\
ECC & $0,5173 \mathrm{~mm}$ & $0,0377 \mathrm{~mm}$ & $0,656 \mathrm{~mm}$ & $0,430 \mathrm{~mm}$ \\
\hline
\end{tabular}

\begin{tabular}{|lcccccc|}
\hline \multicolumn{6}{|c|}{ Tabela 2. Valores médios da ECC nos dois grupos de PIO } \\
PIO & $\mathbf{n}$ & $\begin{array}{c}\text { ECC } \\
\text { média }\end{array}$ & $\begin{array}{c}\text { Desvio } \\
\text { Padrão }\end{array}$ & V. máx. & V. mín. \\
$\leq 21 \mathrm{mmHg}$ & 285 & 0,517 & 0,0376 & 0,660 & 0,430 \\
$>21 \mathrm{mmHg}$ & 34 & 0,519 & 0,0393 & 0,620 & 0,440 \\
\hline
\end{tabular}




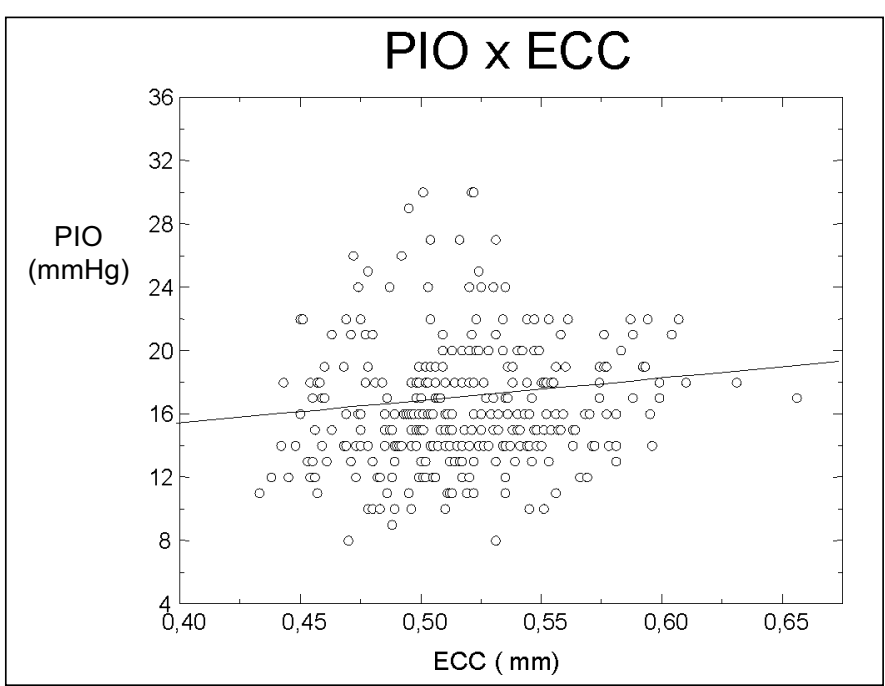

Gráfico 1 - Regressão linear - “ $y=a+b x$ ” $n=319$, com $y=$ P.I.O. "Goldmann"; $x$ = ECC "Topcon". Valor de $a=9,16005 ; b=14,07026$; $r$ (coeficiente de correlação) $=0,13677$ e $p=0,0145$.

dois desvios padrões acima e abaixo deste valor. Portanto o valor limite superior é de $21 \mathrm{mmHg}{ }^{12}$.

O tonômetro de Goldmann é calibrado para medir a PIO em uma espessura corneana central média de $0,54 \mathrm{~mm}$, utilizando-se uma área de aplanação de $3,06 \mathrm{~mm}$. Com estes parâmetros a quantidade de humor aquoso deslocado é de cerca de $0,50 \mathrm{~mm}^{3}$, e a pressão medida no tonômetro é matematicamente semelhante à PIO real (antes do deslocamento) pois tal volume de humor aquoso é praticamente desprezível ${ }^{13}$.

Dentro de variáveis que podem influenciar na medida da PIO, a avaliação da ECC é fundamental ${ }^{12,3}$. Córneas espessas (com fibrilas de colágeno aumentadas), podem resultar em medidas falsamente aumentadas, e córneas delgadas em medidas falsamente diminuídas. Desta forma, considerando um olho com uma PIO verdadeira de $20 \mathrm{mmHg}$, aferida por um catéter intra-ocular acoplado a um manômetro, e com uma ECC de $0,45 \mathrm{~mm}$, ao tonômetro de aplanação apresentará uma PIO de 14,8 mmHg (subestimação da PIO de 5,2 $\mathrm{mmHg}$ ); por outro lado, um olho com uma ECC no valor de $0,59 \mathrm{~mm}$ ao tonômetro de aplanação apresentará uma PIO de $24,7 \mathrm{mmHg}$ (superestimação da PIO de $4,7 \mathrm{mmHg}{ }^{8}$.

Assim, pode-se explicar alguns casos de glaucoma de baixa tensão e hipertensão ocular, baseando-se no erro de estimativa da PIO devido à espessura corneana anormal ${ }^{3,6,8,11,14}$. Isto tem a sua importância nos indivíduos com alta miopia que apresentam uma espessura corneana central menor que indivíduos emétropes ${ }^{16}$ e principalmente, pelo crescente número de pacientes submetidos a cirurgias refrativas, que levam a diminuição da ECC.

Nos resultados encontrados, observou-se uma correlação linear entre PIO e ECC (gráfico I), demonstrando-se, também, a necessidade da avaliação da ECC ao se determinar a PIO do paciente com um método propedêutico básico para o diagnóstico de glaucoma. Portanto, a ECC pode influenciar no resul- tado (valor numérico) da PIO quando se utiliza o tonômetro de aplanação de Goldmann para a sua aferição ${ }^{5-7,14,15}$.

\section{CONCLUSÃO}

Observou-se uma correlação linear entre a pressão intraocular (PIO) e a espessura corneana central (ECC), ou seja, quanto maior a ECC mais alta a PIO.

A ECC aumentada pode levar à aferição de uma PIO erroneamente aumentada através do tonômetro de aplanação de Goldmann. Por outro lado, uma ECC diminuída pode produzir uma subestimação na mensuração da PIO, fato que não deve ser esquecido nos pacientes submetidos a cirurgia refrativa.

A paquimetria corneana deve ser considerada quando da avaliação da necessidade terapêutica para pacientes com PIO alterada mas sem sinais de glaucoma, bem como pacientes com fundo de olho compatível com glaucoma e piora progressiva do campo visual, apresentando PIO em níveis dentro da normalidade.

Portanto, o oftalmologista deve estar atento à medida da ECC, podendo utilizá-la como método propedêutico para avaliação da PIO em casos selecionados.

\section{SUMMARY}

Purpose: To evaluate the distribution of the central corneal thickness (CCT) and its relationship with the intraocular pressure (IOP) in a group of patients and to demonstrate the usefulness of the pachymeter to evaluate IOP in selected cases.

Methods: The central cornea thickness of 167 patients over 40 years old (319 eyes) was determined using ultrasonic pachymetry. The intraocular pressure was measured by the "Goldmann" applanation tonometer.

Results: The CCT average of the 319 eyes was $0.5173+$ $0.0377 \mathrm{~mm}$, the maximum value being $0.656 \mathrm{~mm}$ and the minimum value $0.430 \mathrm{~mm}$. The IOP average was $16.44+$ $3.88 \mathrm{mmHg}$; the maximum pressure $30 \mathrm{mmHg}$ and the minimum pressure $8 \mathrm{mmHg}$. A linear regression of 0.13677 of the analyzed variables $(p=0.0145)$ was observed, with no difference between sex and age. The observations were divided in to two groups: group I-IOP $\leq 21 \mathrm{mmHg}$ - with 285 eyes which presented a CCT average of $0.517+0.0376$ $\mathrm{mm}$; and group II - IOP > $21 \mathrm{mmHg}$ - with 34 eyes which presented a CCT average of $0.519+0.0393 \mathrm{~mm}$.

Conclusion: A linear regression between IOP and CCT was observed, meaning that the higher the CCT the higher the $I O P$ will be. The usefulness of corneal pachymetry for IOP evaluation was shown in those patients where it was falsely increased or decreased on applanation tonometry, directing therapy towards patients really with glaucoma.

Keywords: Intraocular pressure; Central corneal thickness. 


\section{REFERÊNCIAS BIBLIOGRÁFICAS}

1. Shields MB. Glaucoma - A Pressão Intra-Ocular. São Paulo: Panamericana, 1989, p.47-71.

2. Goldmann H, Schmidt TH. Uber applanation-stonometrie. Ophthalmologica 1957; $134: 221$.

3. Ehlers N, Bramsen T, Sperling S. Applanation tonometry and central corneal thickness. Acta Ophthalmol 1975;53:34.

4. Johnson M, Kass MA, Moses RA, Grodzki WJ. Increased corneal thickness simulating elevated intra-ocular pressure. Arch Ophthalmol 1978;96:4,664-5.

5. Wolfs RC, Klaver CC, Vingerling JR, Grobbee DE, Hofman A, de Jong PT Distribution of central corneal thickness and its association with intraocular pressure: The Rotterdam Study. Am J Ophthalmol 1977;123:6,767-72.

5. Herndon LW, Choudhri AS, Cox T, Damji KF, Shields MB, Allingham RR Central corneal thickness in normal, glaucomatous, and ocular hypertensive eyes. Arch Ophthalmol 1997;115:9,1137-4.

6. Stodtmeister R. Applanation tonometry and correction according to corneal thickness. Acta Ophthalmol Scand 1998;76:3,319-24.
8. Whitacre MM, Stein RA, Kassanein K. The effect of corneal thickness on applanation tonometry. Am J Ophthalmol 1993;115:592-6.

9. Hollow FC, Graham PA. Intra-ocular pressure, glaucoma and glaucoma suspects in a defined population. Br J Ophthalmol 1966;50:570-86.

10. Morad Y, Sharon E, Hefetz L, Nemet P. Corneal thickness and curvature in normal-tension glaucoma. Am J Ophthalmol, 1998;125:2,164-8.

11. Mark HH. Corneal curvature in applanation tonometry. Am J Ophthalmol, 1973;76:223

12. Shottenstein, EM. Intra-ocular pressure and tonometry. In: Ritch R, Shields MB, Krupin T. The Glaucomas. St. Louis. $2^{\circ}$ ed., 1989.Vol. I: 20, p.407-445.

13. Stenipak J. Tonometry results using a corneal applanation $3.53 \mathrm{~mm}$ in diameter. Klin Monatsbl Augenheilkd, 1984;184:40.

14. Argus WA. Ocular hypertension and central corneal thickness. Ophthalmology $1995 ; 102: 12,1810-2$.

15. Böhm A, Kohlhaas M, Lerche RC, Bischoff B, Richard G. Measuring intraocular pressure in keratoconus. Effect of the changed biomechanics. Ophthalmology 1997;94:11,771-4.

16. Tanaka HM, Mori ES, Maia NCF, Juliano Y, Campos M. Espessura corneana na alta miopia. Arq Bras Oftalmol 1996;59:3,295-8.

\title{
I CONGRESSO BRASILEIRO DE LENTES DE CONTATO E CÓRNEA
}

\author{
12 a 14 de Abril de 2001 \\ OURO MINAS PALACE HOTEL \\ Belo Horizonte - MG
}

Promoção: Sociedade Brasileira de Lentes de Contato e Córnea - SOBLEC

\author{
Presidente de Honra: Adamo Lui Netto \\ Presidente: Nicomedes Ferreira Filho \\ Secretário: Joel Edmur Boteon \\ Tesoureira: Ana Luiza da S. Galeti Nehemy
}

Informações: Consult Comunicação e Marketing

Tel./Fax: (31) 3274-1550

e-mail:comunica@consultcom.com.br

internet: www.consultcom.com.br 